Please do not remove this page

RMIT

UNIVERSITY

\title{
Computation of thermodynamic and transport properties to predict thermophoretic effects in an argon-krypton mixture
}

Miller, Nicholas; Daivis, Peter; Snook, Ian; Todd, B. D.

https://researchrepository.rmit.edu.au/esploro/outputs/9921858650301341/filesAndLinks?institution=61RMIT_INST\&index=null

Miller, N., Daivis, P., Snook, I., \& Todd, B. D. (2013). Computation of thermodynamic and transport properties to predict thermophoretic effects in an argon-krypton mixture. The Journal of Chemical Physics, 139(14), 1-10. https://doi.org/10.1063/1.4824140

Document Version: Published Version

Published Version: https://doi.org/10.1063/1.4824140

Repository homepage: https://researchrepository.rmit.edu.au

(C) 2013 American Institute of Physics (AIP) Publishing LLC

Downloaded On 2023/04/26 23:30:41 +1000 


\section{DMIT \\ UNIVERSITY}

Thank you for downloading this document from the RMIT Research Repository.

The RMIT Research Repository is an open access database showcasing the research outputs of RMIT University researchers.

RMIT Research Repository: http://researchbank.rmit.edu.au/

\section{Citation:}

Miller, N, Daivis, P, Snook, I and Todd, B 2013, 'Computation of thermodynamic and transport properties to predict thermophoretic effects in an argon-krypton mixture', The Journal of Chemical Physics, vol. 139, no. 14, 144504, pp. 1-10.

See this record in the RMIT Research Repository at:

http://researchbank.rmit.edu.au/view/rmit:22507

Version: Published Version

Copyright Statement: (c) 2013 AIP Publishing LLC

Link to Published Version:

http://dx.doi.org/10.1063/1.4824140

\section{PLEASE DO NOT REMOVE THIS PAGE}




\title{
Computation of thermodynamic and transport properties to predict thermophoretic effects in an argon-krypton mixture
}

\author{
Nicholas A. T. Miller, ${ }^{1}$ Peter J. Daivis, ${ }^{1, a)}$ Ian K. Snook, ${ }^{1, b)}$ and B. D. Todd ${ }^{2}$ \\ ${ }^{1}$ School of Applied Sciences, RMIT University, GPO Box 2476, Melbourne, Victoria 3001, Australia \\ ${ }^{2}$ Mathematics, Faculty of Engineering and Industrial Sciences, and Centre for Molecular Simulation, \\ Swinburne University of Technology, PO Box 218, Hawthorn, Victoria 3122, Australia
}

(Received 12 August 2013; accepted 19 September 2013; published online 10 October 2013)

\begin{abstract}
Thermophoresis is the movement of molecules caused by a temperature gradient. Here we report the results of a study of thermophoresis using non-equilibrium molecular dynamics simulations of a confined argon-krypton fluid subject to two different temperatures at thermostated walls. The resulting temperature profile between the walls is used along with the Soret coefficient to predict the concentration profile that develops across the channel. We obtain the Soret coefficient by calculating the mutual diffusion and thermal diffusion coefficients. We report an appropriate method for calculating the transport coefficients for binary systems, using the Green-Kubo integrals and radial distribution functions obtained from equilibrium molecular dynamics simulations of the bulk fluid. Our method has the unique advantage of separating the mutual diffusion and thermal diffusion coefficients, and calculating the sign and magnitude of their individual contributions to thermophoresis in binary mixtures. (C) 2013 AIP Publishing LLC. [http://dx.doi.org/10.1063/1.4824140]
\end{abstract}

\section{INTRODUCTION}

In this study, we predict the concentration profile of a binary fluid under non-equilibrium conditions. We compute the transport properties of a two component fluid at equilibrium, and use these properties to predict the concentration profile induced by a temperature gradient, applied by maintaining parallel confining walls at different temperatures.

Thermal diffusion exists because a temperature gradient applied to a mixture of two components will give rise to a gradient in the concentration of these components. ${ }^{1}$ Modelling fluid flow with confining planar walls provides a simplified geometry to analyse the effects of thermophoresis. Early techniques used thermal diffusion for isotope separation. Clusius and Dickel ${ }^{2}$ built the first thermal diffusion column, and obtained a separation factor, $q$, for $\mathrm{HCl}^{35}$ and $\mathrm{HCl}^{37}$ of 1.01 . This compared favourably to the separation factors for distillation, chemical exchange, and centrifugal techniques of the time. Recent experiments have extended the understanding of thermophoresis in this geometry to a number of liquid mixtures including toluene with n-hexane, carbon tetrachloride with cyclohexane, and methanol with ethanol. ${ }^{3}$

The importance of understanding thermal diffusion and the current lack of knowledge of the process in liquid mixtures is highlighted by Wiegand. ${ }^{4}$ Furthermore, the importance of understanding thermophoresis in colloidal suspensions is highlighted by Piazza and Parola, who emphasise its role as a tool for particle manipulation in fluidic systems. ${ }^{5}$ Our study furthers the understanding of the effects of thermal diffusion in binary fluids and the contributions to thermophoresis from the mutual and thermal diffusion coefficients.

\footnotetext{
a)Electronic mail: peter.daivis@rmit.edu.au

b) Deceased.
}

Argon-krypton (Ar-Kr) is a well studied two component mixture partly due to the inert nature of the components. Studying this fluid provides the opportunity to expand upon current knowledge of two component fluidic systems, and test the method presented in this study. By validating the transport coefficients we obtain with results in the literature, and with the successful prediction of concentration profiles using a continuum mechanics description that relies on these coefficients, we provide a framework for future research into the transport mechanisms of binary systems.

Previous work presented in the literature highlights two major issues when calculating the transport coefficients for two component systems. The first is the large spatial range over which the radial distribution function, $g_{a b}(r)$, decays. It is important to compute correct values for the three $g_{a b}(r)$ functions to calculate an accurate value of the thermodynamic factor, $\partial \mu_{k} / \partial c_{k}$, required for calculating the mutual diffusion coefficient, $D_{m}$. This issue has been discussed in detail by several authors. ${ }^{6,7}$ We apply Kirkwood-Buff theory directly by fitting the tail of the radial distribution function using a method similar to that used by Gaylor, Snook, and van Megen to fit structure factors obtained by Fourier transformation of radial distribution factors. ${ }^{8}$

Ideality has frequently been assumed when calculating the mutual diffusion coefficient for Ar-Kr fluids, beginning with the work by Jolly and Bearman. ${ }^{9}$ Inaccurate values of the thermodynamic factor were also obtained by Schoen and Hoheisel, ${ }^{10}$ and they did not include errors in their reported value due to the inability to obtain $g_{a b}(r)$ at large $r$. An analytical expression for calculating $\partial \mu_{k} / \partial c_{k}$ for ideal fluids ${ }^{11}$ was used to calculate the Soret coefficient, $S_{T}$ for an $\mathrm{Ar}-\mathrm{Kr}$ mixture assuming ideality by Perronance et al. ${ }^{12}$ Nichols, Moore, and Wheeler noted that truncation of the radial distribution function biases the evaluation of the thermodynamic factor towards the result for an ideal gas mixture. ${ }^{6}$ Consequently, 
the error in the thermodynamic factor was not fully appreciated in early work. We find that the Ar-Kr fluid studied here is far from ideal, and that the assumption of ideality can lead to inaccurate values of $\partial \mu_{k} / \partial c_{k}$, and subsequently $D_{m}$.

The second issue is that the practical transport coefficients depend on the so-called primed phenomenological coefficients, whereas it is the unprimed ones that are obtained from the Green-Kubo (G-K) time correlation functions. The primed phenomenological coefficients are defined with respect to one set of thermodynamic fluxes and forces which are convenient for measurement, whereas the unprimed ones are defined with respect to another set, which are more convenient for computation. In previous work on Ar- $\mathrm{Kr}$ mixtures, this distinction has led to computations only of the relevant $\mathrm{G}-\mathrm{K}$ integrals and unprimed phenomenological coefficients and not the transport coefficients. ${ }^{13,14}$ However, the primed coefficients can easily be expressed as a combination of the unprimed ones. The appropriate combinations are presented by de Groot and Mazur, ${ }^{11}$ and similar ones are utilised by Galamba, Nieto de Castro, and Ely for calculating the thermal conductivity of molten alkali halides. ${ }^{15}$

The method presented here to overcome these issues when calculating transport coefficients for two component systems is validated with a comparison of the thermal diffusion factor $\alpha_{12}$ obtained by Hafskjold, Ikeshoji, and Ratkje. ${ }^{16}$ In their work a heat boundary-driven non-equilibrium molecular dynamics algorithm is used to calculate $\alpha_{12}$ for an equimolar Ar-Kr fluid, and we obtain a value that agrees with theirs. Our method has the advantage of separating the mutual diffusion and thermal diffusion coefficients.

\section{THEORY}

\section{A. Relation between primed and unprimed phenomenological coefficients}

With the introduction of a second species in a binary fluid, the diffusion and heat flux both need to be considered. Diffusion is driven by the gradients of chemical potential and temperature, which can be expressed in terms of the concentration and temperature gradients. With component 1 as the solute, consisting of the larger particles $(\mathrm{Kr})$ and 2 as the solvent (Ar), the equation for the mass flux of component 1 can be defined as ${ }^{11}$

$$
\mathbf{J}_{1}=-\frac{L_{11}^{\prime}}{T}\left[\frac{1}{c_{2}} \frac{\partial \mu_{1}}{\partial c_{1}} \nabla c_{1}\right]_{p, T}-\frac{L_{1 q}^{\prime}}{T^{2}} \nabla T
$$

and the heat flux is defined as

$$
\mathbf{J}_{q}^{\prime}=-\frac{L_{q q}^{\prime}}{T^{2}} \nabla T-\frac{L_{q 1}^{\prime}}{T}\left[\frac{1}{c_{2}} \frac{\partial \mu_{1}}{\partial c_{1}} \nabla c_{1}\right]_{p, T} .
$$

In these two equations, $T$ is the temperature and $c_{k}$ is the mass fraction of component $k$, defined as the ratio of the component's mass density $\rho_{k}$ to the total mass density of the solution $\rho . \partial \mu_{k} / \partial c_{k}$ is the thermodynamic factor and $p$ is the pressure. Importantly $L_{a b}^{\prime}$ are combinations of the unprimed phenomenological coefficients, which are obtained from the usual G-K time correlation functions ${ }^{17}$

$$
\begin{gathered}
L_{a b}=\lim _{t \rightarrow \infty} L_{a b}(t), \\
L_{a b}(t)=\int_{0}^{t} C_{a b}\left(t^{\prime}\right) d t^{\prime}, \\
C_{a b}(t)=\frac{V}{3 k_{B}}\left\langle\mathbf{J}_{a}(t) \cdot \mathbf{J}_{b}(0)\right\rangle .
\end{gathered}
$$

These equations are used to calculate the mass-mass phenomenological coefficient $L_{11}$, the heat-heat phenomenological coefficient $L_{q q}$, and the mass-heat and heat-mass crosscorrelated phenomenological coefficients, $L_{q 1}$ and $L_{1 q}$, respectively, for the Ar-Kr fluid. Due to the Onsager reciprocal relations the cross-correlated phenomenological coefficients are equal, $L_{1 q}=L_{q 1}$. We show later in Eqs. (31)-(34) how the combinations of the phenomenological coefficients should be used for two component fluids to obtain the primed phemenological coefficients $L_{a b}^{\prime}$.

The mass flux due to a temperature gradient is called the Soret effect, and the heat flux due to a concentration gradient is called the Dufour effect. From the constitutive relations ${ }^{11}$

$$
\mathbf{J}_{1}=-\rho D_{m} \nabla c_{1}-\rho c_{1} c_{2} D^{\prime} \nabla T
$$

and

$$
\mathbf{J}_{q}^{\prime}=-\lambda \nabla T-\rho_{1} \frac{\partial \mu_{1}}{\partial c_{1}} T D^{\prime \prime} \nabla c_{1},
$$

we define the practical transport coefficients being the thermal conductivity, $\lambda$, the mutual diffusion coefficient, $D_{m}$, the thermal diffusion coefficient, $D^{\prime}$, and the Dufour coefficient, $D^{\prime \prime}$. These transport coefficients are expressed in terms of the phenomenological coefficients as ${ }^{11}$

$$
\begin{gathered}
\lambda=\frac{L_{q q}^{\prime}}{T^{2}}, \\
D_{m}=\frac{L_{11}^{\prime}}{\rho c_{2} T}\left(\frac{\partial \mu_{1}}{\partial c_{1}}\right)_{p, T}, \\
D^{\prime}=\frac{L_{1 q}^{\prime}}{\rho c_{1} c_{2} T^{2}},
\end{gathered}
$$

and

$$
D^{\prime \prime}=\frac{L_{q 1}^{\prime}}{\rho c_{1} c_{2} T^{2}} .
$$

To obtain the phenomenological coefficients the integrals of the equilibrium G-K time correlation functions are used. In binary systems, the phenomenological coefficients in Eqs. (8)(11) are a combination of the usual phenomenological coefficients obtained from the G-K integrals.

The entropy production for a binary fluid, that has no external fields or viscous flow, with temperature and concentration gradients is given by ${ }^{11}$

$$
\sigma=-\frac{1}{T^{2}} \mathbf{J}_{q} \cdot \nabla T-\sum_{k=1}^{2} \mathbf{J}_{k} \cdot \nabla\left(\frac{\mu_{k}}{T}\right) .
$$

The chemical potential gradient can be decomposed into the concentration and temperature dependent parts at constant 
pressure as

$$
\nabla \mu_{k}=\frac{\mu_{k}-h_{k}}{T} \nabla T+\left(\nabla \mu_{k}\right)_{T, p} .
$$

Here $h_{k}$ is the partial specific enthalpy of component $k$. Substituting Eq. (13) into the equation for entropy production we obtain

$$
\sigma=-\frac{1}{T^{2}} \mathbf{J}_{q} \cdot \nabla T-\sum_{k=1}^{2} \mathbf{J}_{k} \cdot\left(\frac{1}{T}\left(\nabla \mu_{k}\right)_{T, p}-\frac{h_{k}}{T^{2}} \nabla T\right) .
$$

The Gibbs-Duhem equation for the chemical potential gradients at constant temperature and pressure gives ${ }^{11}$

$$
\sum_{k=1}^{r} c_{k}\left(\nabla \mu_{k}\right)_{T, p}=0
$$

and the diffusive fluxes satisfy

$$
\begin{aligned}
\sum_{k=1}^{2} \mathbf{J}_{k} \cdot\left(\nabla \mu_{k}\right)_{T, p} & =\mathbf{J}_{1} \cdot\left(\nabla \mu_{1}\right)_{T, p}+\frac{c_{1}}{c_{2}} \mathbf{J}_{1} \cdot\left(\nabla \mu_{1}\right)_{T, p} \\
& =\left(1+\frac{c_{1}}{c_{2}}\right) \mathbf{J}_{1} \cdot\left(\nabla \mu_{1}\right)_{T, p} .
\end{aligned}
$$

The entropy production in Eq. (14) can now be written as

$$
\begin{aligned}
\sigma= & -\frac{1}{T^{2}} \mathbf{J}_{q} \cdot \nabla T \\
& -\frac{1}{T} \mathbf{J}_{1} \cdot\left[\left(1+\frac{c_{1}}{c_{2}}\right)\left(\nabla \mu_{k}\right)_{T, p}-\frac{1}{T}\left(h_{1}-h_{2}\right) \nabla T\right] .
\end{aligned}
$$

The entropy production can also be written with the fluxes left in their original form and the thermodynamic forces written as

$$
\begin{gathered}
\mathbf{X}_{q}=\left(\frac{\nabla T}{T^{2}}\right), \\
\mathbf{X}_{1}=\frac{1}{T}\left[\left(1+\frac{c_{1}}{c_{2}}\right)\left(\nabla \mu_{k}\right)_{T, p}-\frac{1}{T}\left(h_{1}-h_{2}\right) \nabla T\right],
\end{gathered}
$$

resulting in the entropy production written in the form

$$
\sigma=-\mathbf{J}_{q} \cdot \mathbf{X}_{q}-\mathbf{J}_{1} \cdot \mathbf{X}_{1}
$$

Now we introduce the primed heat flux into the entropy production

$$
\begin{aligned}
\sigma= & -\frac{1}{T^{2}}\left(\mathbf{J}_{q}-\sum_{k=1}^{2} h_{k} \mathbf{J}_{k}\right) \cdot \nabla T \\
& -\frac{1}{T} \mathbf{J}_{1} \cdot\left(1+\frac{c_{1}}{c_{2}}\right)\left(\nabla \mu_{1}\right)_{T, p}
\end{aligned}
$$

or

$$
\sigma=-\mathbf{J}_{q}^{\prime} \cdot \mathbf{X}_{q}-\mathbf{J}_{1} \cdot \mathbf{X}_{1}^{\prime} \text {. }
$$

The difference between $\mathbf{J}_{q}^{\prime}$ and $\mathbf{J}_{q}$ represents the transfer of heat due to diffusion. ${ }^{11}$ The linear phenomenological equations for the unprimed variables are defined for the heat flux and mass flux as ${ }^{11}$

$$
\mathbf{J}_{q}=-L_{q q} \mathbf{X}_{q}-L_{q 1} \mathbf{X}_{1}
$$

and

$$
\mathbf{J}_{1}=-L_{11} \mathbf{X}_{1}-L_{1 q} \mathbf{X}_{q},
$$

and for the primed variables

$$
\mathbf{J}_{q}^{\prime}=-L_{q q}^{\prime} \mathbf{X}_{q}-L_{q 1}^{\prime} \mathbf{X}_{1}^{\prime}
$$

and

$$
\mathbf{J}_{1}^{\prime}=-L_{11}^{\prime} \mathbf{X}_{1}^{\prime}-L_{1 q}^{\prime} \mathbf{X}_{q} .
$$

In this notation, Eq. (19) becomes

$$
\mathbf{X}_{1}=\mathbf{X}_{1}^{\prime}-\left(h_{1}-h_{2}\right) \mathbf{X}_{q} .
$$

Now the entropy production can be written in terms of the primed flux phenomenological coefficients as

$$
\sigma=L_{q q}^{\prime} \mathbf{X}_{q} \cdot \mathbf{X}_{q}+L_{q 1}^{\prime} \mathbf{X}_{1}^{\prime} \cdot \mathbf{X}_{q}+L_{11}^{\prime} \mathbf{X}_{1}^{\prime} \cdot \mathbf{X}_{1}^{\prime}+L_{1 q}^{\prime} \mathbf{X}_{q} \cdot \mathbf{X}_{1}^{\prime},
$$

and for the unprimed variables the entropy production is

$$
\sigma=L_{q q} \mathbf{X}_{q} \cdot \mathbf{X}_{q}+L_{q 1} \mathbf{X}_{1} \cdot \mathbf{X}_{q}+L_{11} \mathbf{X}_{1} \cdot \mathbf{X}_{1}+L_{1 q} \mathbf{X}_{q} \cdot \mathbf{X}_{1} \cdot
$$

If we substitute the primed thermodynamic forces into Eq. (29), we obtain

$$
\begin{aligned}
\sigma= & L_{q q} \mathbf{X}_{q} \cdot \mathbf{X}_{q}+L_{q 1}\left(\mathbf{X}_{1}^{\prime}-\left(h_{1}-h_{2}\right) \mathbf{X}_{q}\right) \cdot \mathbf{X}_{q} \\
& +L_{11}\left(\mathbf{X}_{1}^{\prime}-\left(h_{1}-h_{2}\right) \mathbf{X}_{q}\right)^{2} \\
& +L_{1 q} \mathbf{X}_{q} \cdot\left(\mathbf{X}_{1}^{\prime}-\left(h_{1}-h_{2}\right) \mathbf{X}_{q}\right) .
\end{aligned}
$$

Comparing this with the entropy production in Eq. (28), we find

$$
\begin{gathered}
L_{q q}^{\prime}=L_{q q}-L_{q 1}\left(h_{1}-h_{2}\right)+L_{11}\left(h_{1}-h_{2}\right)^{2} \\
-L_{1 q}\left(h_{1}-h_{2}\right), \\
L_{q 1}^{\prime}=L_{q 1}-L_{11}\left(h_{1}-h_{2}\right), \\
L_{11}^{\prime}=L_{11},
\end{gathered}
$$

and

$$
L_{1 q}^{\prime}=L_{q 1}-L_{11}\left(h_{1}-h_{2}\right) .
$$

The Onsager reciprocal relations still hold in this case, therefore $L_{1 q}^{\prime}=L_{q 1}^{\prime}$. It can be seen in Eqs. (31)-(34) that the difference in the partial specific enthalpies $\left(h_{1}-h_{2}\right)$ is required.

\section{B. Calculation of thermodynamic properties}

To obtain the partial specific enthalpy of component $k$, we use the relation

$$
h_{k}=\left(\frac{\partial H}{\partial M_{k}}\right)_{T, p,\left\{M_{i \neq k}\right\}},
$$

which are obtained for the $\mathrm{Kr}$ and Ar species by holding the number of either $\mathrm{Kr}$ or Ar particles constant while varying the number of $\mathrm{Ar}$ or $\mathrm{Kr}$ particles, respectively. In Eq. (35), $M_{k}$ is the total mass of components of type $k$, and the enthalpy $H$, is calculated for each system through the usual relation

$$
H=U+p V .
$$


Here $U$ is the total internal energy, and $V$ the total volume of the fluid.

By expressing the primed phenomenological coefficients in terms of the unprimed coefficients, we can fully account for the effects of heat conduction and diffusion in binary mixtures. This allows us to obtain accurate values for the transport coefficients of Eqs. (8)-(11) for a binary fluid. The importance of the partial enthalpies in the calculation of $D_{T}$ was recognised by Vogelsang et al. ${ }^{18}$ who used a single simulation to calculate partial enthalpies and volumes using a method introduced by Sindzingre, Ciccotti, and Massobrio. ${ }^{19}$ However, they did not use Eqs. (31)-(34) to calculate the transport coefficients.

Also required in the calculation of the mutual diffusion coefficient, $D_{m}$, is the thermodynamic factor. This is calculated using the theory presented by Kirkwood and Buff ${ }^{20}$ on the relation between the thermodynamic factor and $G_{a b}$, the volume integral of the radial distribution function,

$$
G_{a b}=\int\left(g_{a b}(r)-1\right) d V .
$$

The relation of Eq. (37) to the thermodynamic factor for component 1 is given by

$$
\left(\frac{\partial \mu_{1}}{\partial c_{1}}\right)_{p, T}=k_{B} T \rho_{m}\left(\frac{G_{12}-G_{11}}{1+c_{1}\left(G_{11}-G_{12}\right)}\right) .
$$

Here $k_{B}$ is Boltzmann's constant. Because the pair distribution decays over a large spatial distance and the pair distribution can only be computed to half the box length of the bulk fluid, we require a fit to the tail of the radial distribution function so the remaining decay can be integrated over all space. We have used a function of the form

$$
g_{a b}(r)=1+A \cdot e^{-B r} \cdot \cos (C r+D),
$$

where $A, B, C$, and $D$ are fitting parameters chosen to minimise the residual of the fit to the data in the tail of the simulated radial distribution function. This is a modified form of the function used by Gaylor, Snook and van Megen to fit radial distribution functions and obtain structure factors by Fourier transformation. ${ }^{8}$ We note that the radial distribution function calculated in the canonical ensemble, ${ }^{21}$ does not converge to 1 but rather to $1-k / N$, where $N$ is the total number of particles and $k$ is some constant. The size of the system used to calculate the pair distribution is chosen such that $k / N$ is smaller than the error in the fit used to force the convergence to 1 . This issue could potentially be avoided with a recent development by Krüger et al. who present a method to calculate the exact expressions for finite volume Kirkwood-Buff integrals. $^{7}$

To provide a more complete set of transport coefficients for the equimolar Ar-Kr fluid we also report the value of the viscosity, $\eta$. The viscosity is calculated from the stress autocorrelation function, and for a homogeneous and isotropic fluid at equilibrium, the symmetry of the material can be used. The zero strain rate viscosity is calculated by averaging over the elements of the symmetric traceless pressure tensor. As reported by Daivis and Evans ${ }^{22}$

$$
\eta=\frac{V}{10 k_{B} T} \int_{0}^{\infty}\left\langle\mathbf{P}^{t s}(t): \mathbf{P}^{t s}(0)\right\rangle d t .
$$

This results from the fact that there are only five independent components of the traceless symmetric part of the stress tensor. Using the sum of five different correlation functions increases the statistical reliability of the viscosity computation yet maintains computational efficiency. ${ }^{22}$

\section{Confined system profile predictions}

The relationship between the steady state concentration and temperature gradients is derived from the linear constitutive relations. In the steady state, the diffusive fluxes are zero

$$
\mathbf{J}_{1}=-\rho D_{m} \nabla c_{1}-\rho c_{1} c_{2} D^{\prime} \nabla T=0
$$

or, assuming a planar geometry,

$$
D_{m} \frac{\partial c_{1}}{\partial y}+c_{1} c_{2} D^{\prime} \frac{\partial T}{\partial y}=0 .
$$

Integrating this equation to obtain the concentration profile results in the relation

$$
c(y)=\frac{1}{\frac{1-c_{0}}{c_{0}} \exp \left(\frac{D^{\prime}}{D_{m}}\left(T(y)-T_{0}\right)\right)+1},
$$

where $c_{0}$ and $T_{0}$ are the concentration and temperature at some reference point in the system. The thermal diffusion and mutual diffusion coefficients are required in the form of their ratio, often denoted as the Soret coefficient, $S_{T}$, in Eq. (43). Thermophoresis, also known as the Soret effect, or thermal diffusion, causes the migration of particles along temperature gradients. The effect is studied experimentally for moderate thermal gradients applied in a mixture of $200 \mathrm{~nm}$ polystyrene spheres in water by Duhr and Braun. ${ }^{23}$ They report a thermophoretic depletion in the region of elevated temperature. The authors have also demonstrated the ability to utilise thermophoresis and fluid flow for trapping of small biomolecules. ${ }^{24}$ In this study, they observe an increased concentration in the heated region. The direction of migration is dependent on the sign of $S_{T}$. The method presented in our work has the advantage of determining the magnitude of the transport coefficients $D_{m}$ and $D^{\prime}$, the sign of $D^{\prime}$, and how each contributes to thermophoresis.

\section{METHOD}

\section{A. System parameters}

To test the theory outlined in Sec. II A, we simulated an equimolar Ar-Kr system using the Lennard-Jones parameters in Table I. Reducing with respect to the Ar-Ar parameters, and using $r_{c}=2.5 \sigma_{i j}$ for the cut-off of the Lennard-Jones (L-J) potential, the simulation parameters in reduced units are presented in Table II. The L-J potential takes the usual form

$$
\phi(r)^{L J}=4 \epsilon\left[\left(\frac{\sigma}{r}\right)^{12}-\left(\frac{\sigma}{r}\right)^{6}\right],
$$

where $r$ is the distance between the particles, $\epsilon$ is the depth of the potential well, and $\sigma$ is the distance at which the potential is zero. For the equimolar Ar- $\mathrm{Kr}$ fluid studied here the L-J 
TABLE I. Argon-krypton simulation Lennard-Jones parameters.

\begin{tabular}{lcccc}
\hline \hline Species & Index & $\epsilon / 10^{-21} \mathrm{~J}$ & $\sigma / 10^{-10} \mathrm{~m}$ & $m / 10^{-26} \mathrm{~kg}$ \\
\hline $\mathrm{Ar}-\mathrm{Ar}$ & $2-2$ & 1.654 & 3.405 & 6.634 \\
$\mathrm{Kr}-\mathrm{Kr}$ & $1-1$ & 2.305 & 3.663 & 13.915 \\
$\mathrm{Ar}-\mathrm{Kr}$ & $2-1$ & 1.952 & 3.519 & \\
\hline \hline
\end{tabular}

potential is shifted and truncated

$$
\phi(r)= \begin{cases}4 \epsilon\left[\left(\frac{\sigma}{r}\right)^{12}-\left(\frac{\sigma}{r}\right)^{6}\right]-\phi_{c}^{L J} & r \leq r_{c} \\ 0 & r>r_{c} .\end{cases}
$$

The state point was chosen to match that studied by Hafskjold, Ikeshoji, and Ratkje, ${ }^{16}$ the number density, $n$ is 0.7138 and $T$ is 0.965 . To calculate the G-K integrals and the radial distribution functions, systems composed of 864 particles were used. The G-K integral data was ensemble averaged over 32 macroscopically similar systems, varied by the application of 32 slightly different temperatures through a Gaussian thermostat, then re-equilibrated to the temperature of 0.965 before accumulating the correlation functions. To obtain the partial specific enthalpy of the $\mathrm{Kr}$ species, 8 separate systems were simulated, composed of $848,852,856,860,868,872$, 876, and $880 \mathrm{Kr}$ particles, all with $864 \mathrm{Ar}$ particles. To obtain the partial specific enthalpy of the Ar species, the same range of $848-880$ particles were used this time for the $\mathrm{Ar}$ species, with $864 \mathrm{Kr}$ particles. For the bulk fluid simulations, periodic boundary conditions were used in all three Cartesian directions.

\section{RESULTS AND DISCUSSION}

\section{A. Bulk fluid}

\section{Green-Kubo time correlation functions}

The G-K time correlation functions are shown in Figs. 1-5. The zoomed boxes highlight the quick decay of the correlation functions to zero. The integrals can be seen to have converged as there are no significant deviations or fluctuations in the integrals. The values of the G-K integrals are extracted after the convergence. The value of the integral of the mass flux auto-correlation function obtained for the Ar- $\mathrm{Kr}$ fluid is $-0.01692(7)$. Knowing that the sum of the diffusive fluxes is zero in our simulations and $\mathbf{J}_{1}=-\mathbf{J}_{2}$, this result is comparable with the value of $0.0174(5)$ obtained by Sarman and Evans. ${ }^{14}$

For the integral of the heat flux auto-correlation function, we obtained a value of 4.14(2). The value we obtained is comparable to the result of Sarman and Evans ${ }^{14}$ who report a value of $4.12(2)$.

TABLE II. Reduced argon-krypton Lennard-Jones parameters.

\begin{tabular}{llllll}
\hline \hline Interaction & Index & \multicolumn{1}{c}{$\epsilon$} & \multicolumn{1}{c}{$\sigma$} & $m$ & $r_{c}$ \\
\hline $\mathrm{Ar}-\mathrm{Ar}$ & $2-2$ & 1 & 1 & 1 & 2.5 \\
$\mathrm{Kr}-\mathrm{Kr}$ & $1-1$ & 1.39384 & 1.06696 & 2.0976 & 2.6674 \\
$\mathrm{Ar}-\mathrm{Kr}$ & $2-1$ & 1.180612 & 1.03348 & & 2.5837 \\
\hline \hline
\end{tabular}
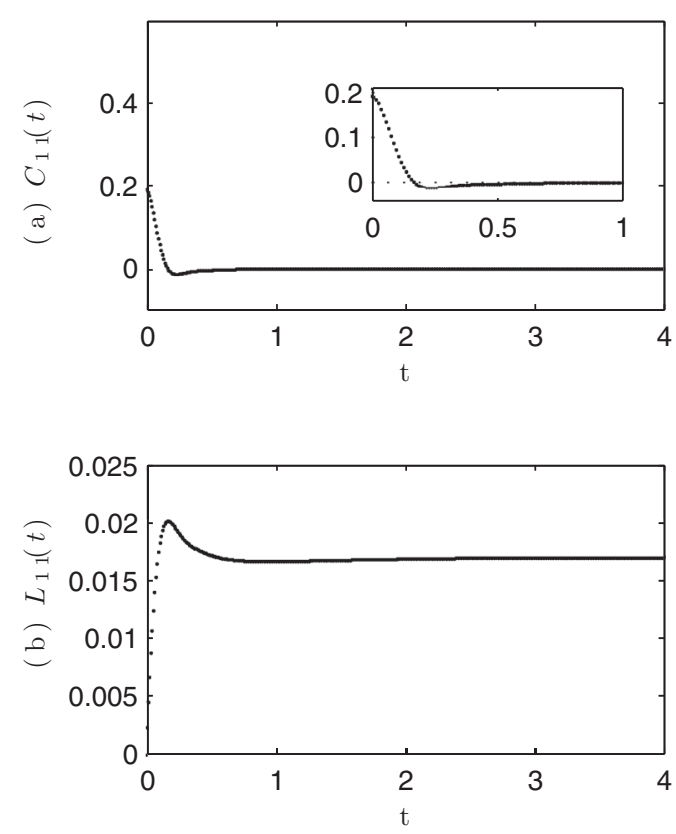

FIG. 1. Mass flux (a) auto-correlation function and (b) its integral for equimolar $\mathrm{Ar}-\mathrm{Kr}, n=0.7138$ and $T=0.965$.

For the average of the integrals of the cross-correlation functions, we have computed a value of $-0.0171(1)$. This is in excellent agreement again with Sarman and Evans ${ }^{14}$ who obtained a value of $-0.0175(8)$, and MacGowan and Evans ${ }^{13}$ who obtained a value of $-0.0162(50)$.

The stress auto-correlation function and its integral are shown in Fig. 6. The value of the integral of the stress autocorrelation function was calculated using Eq. (40) and the value of $\eta$ we obtained is 2.491(8). To our knowledge at the time of the submission of this publication there were no reported values of the viscosity in the literature for equimolar $\mathrm{Ar}-\mathrm{Kr}$ at the state point studied here.
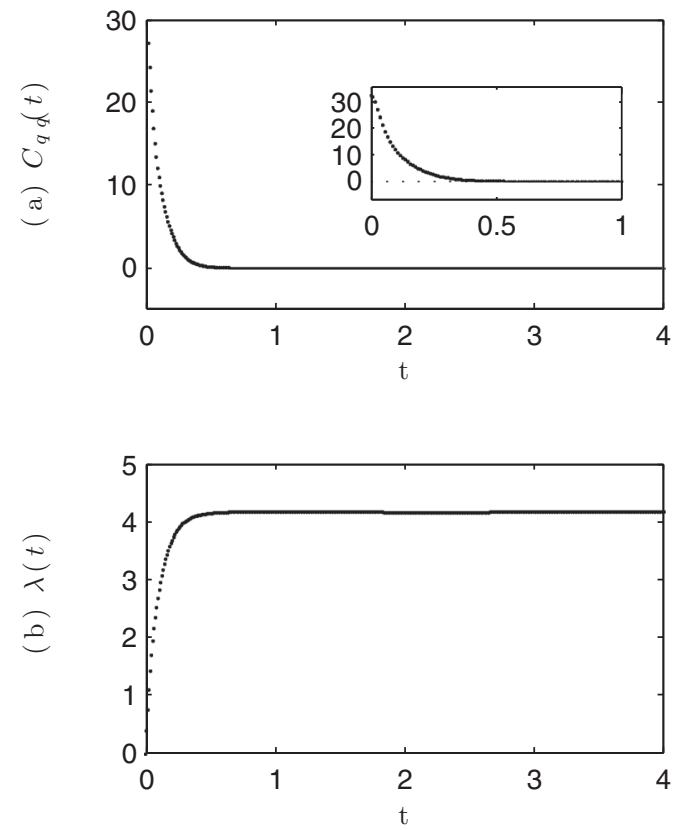

FIG. 2. Heat flux (a) auto-correlation function and (b) its integral for equimolar Ar-Kr, $n=0.7138$ and $T=0.965$. 

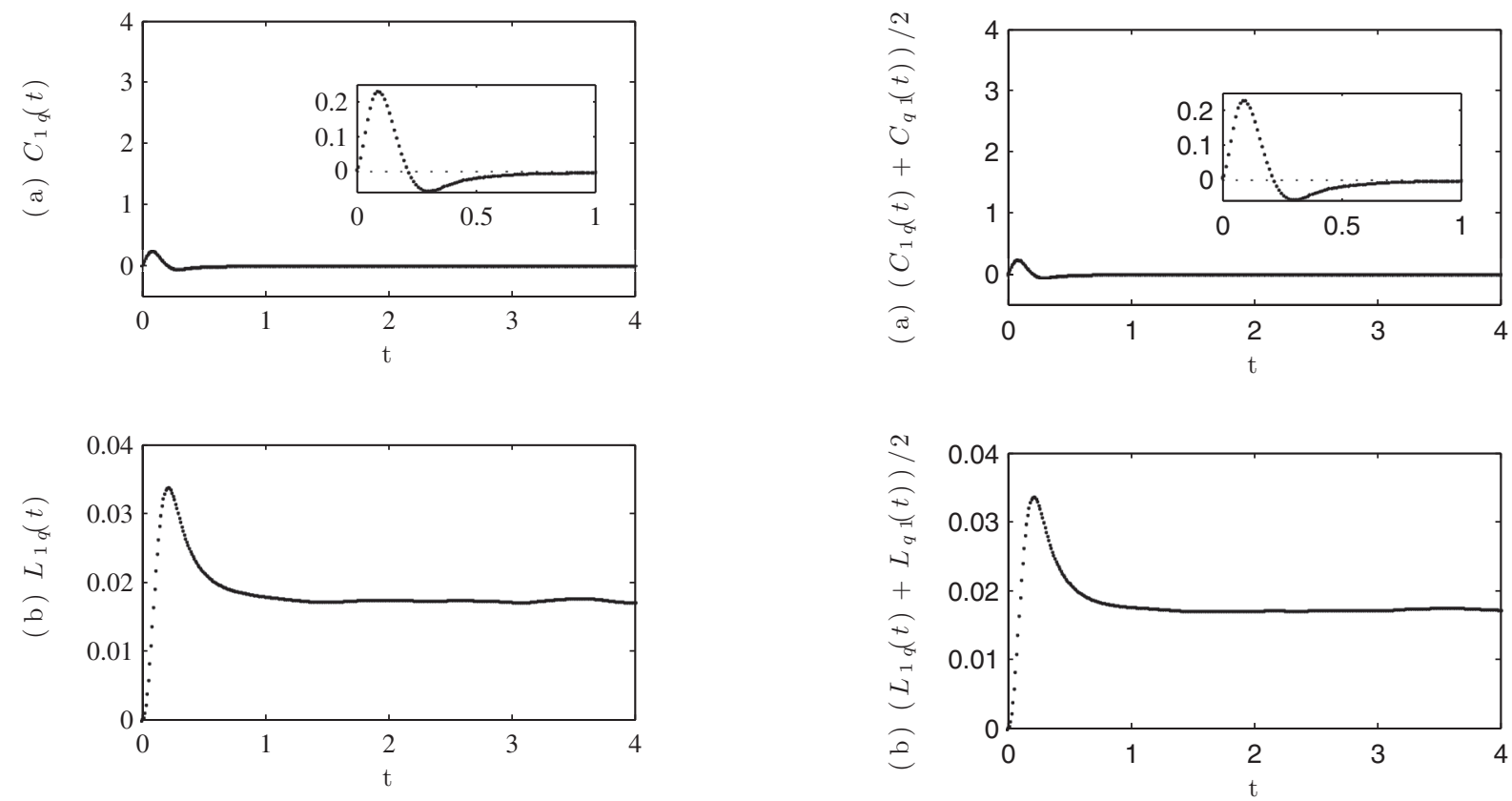

FIG. 3. Mass-heat (a) cross-correlation function and (b) its integral for equimolar Ar-Kr, $n=0.7138$ and $T=0.965$.

FIG. 5. Average of heat-mass and mass-heat (a) cross-correlation functions and (b) their integrals for equimolar Ar-Kr, $n=0.7138$ and $T=0.965$.

\section{Radial distribution functions}

To obtain the radial distribution functions for equimolar Ar-Kr, the volume integrals have been calculated using Eq. (37) and are shown in Figs. 7-9. These figures show (a) the radial distribution function for the relevant species and their fit from Eq. (39), (b) the integral of the pair distribution and their fit, and (c) the residual of the pair distribution and the fit of the radial distribution function. The residuals are of the order of $10^{-3}$ indicating a good fit for the spatial decay in the pair distributions. The values of the volume integrals
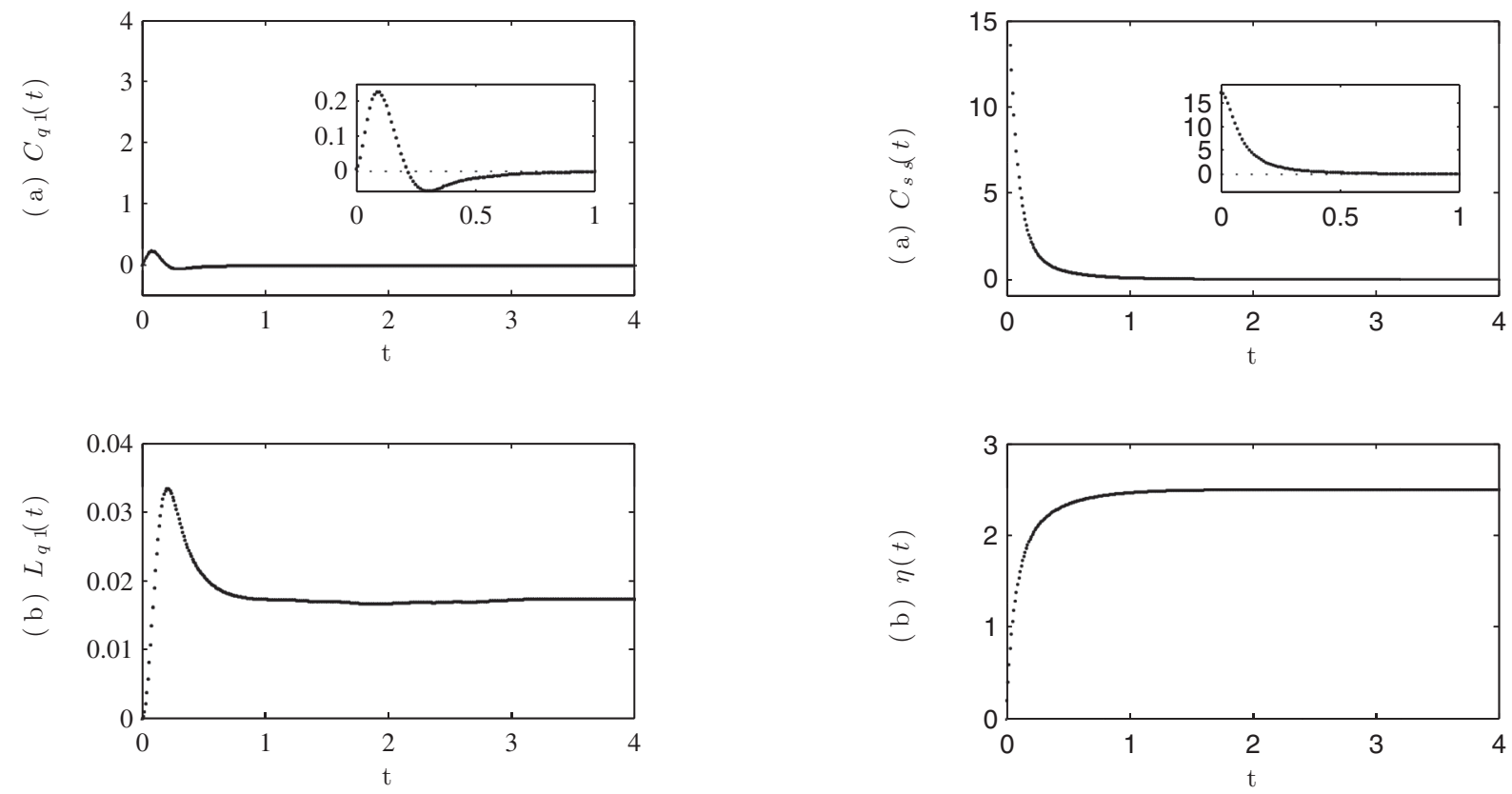

FIG. 4. Heat-mass (a) cross-correlation function and (b) its integral for equimolar Ar-Kr, $n=0.7138$ and $T=0.965$.

FIG. 6. Stress (a) auto-correlation function and (b) its integral for equimolar Ar-Kr, $n=0.7138$ and $T=0.965$. 

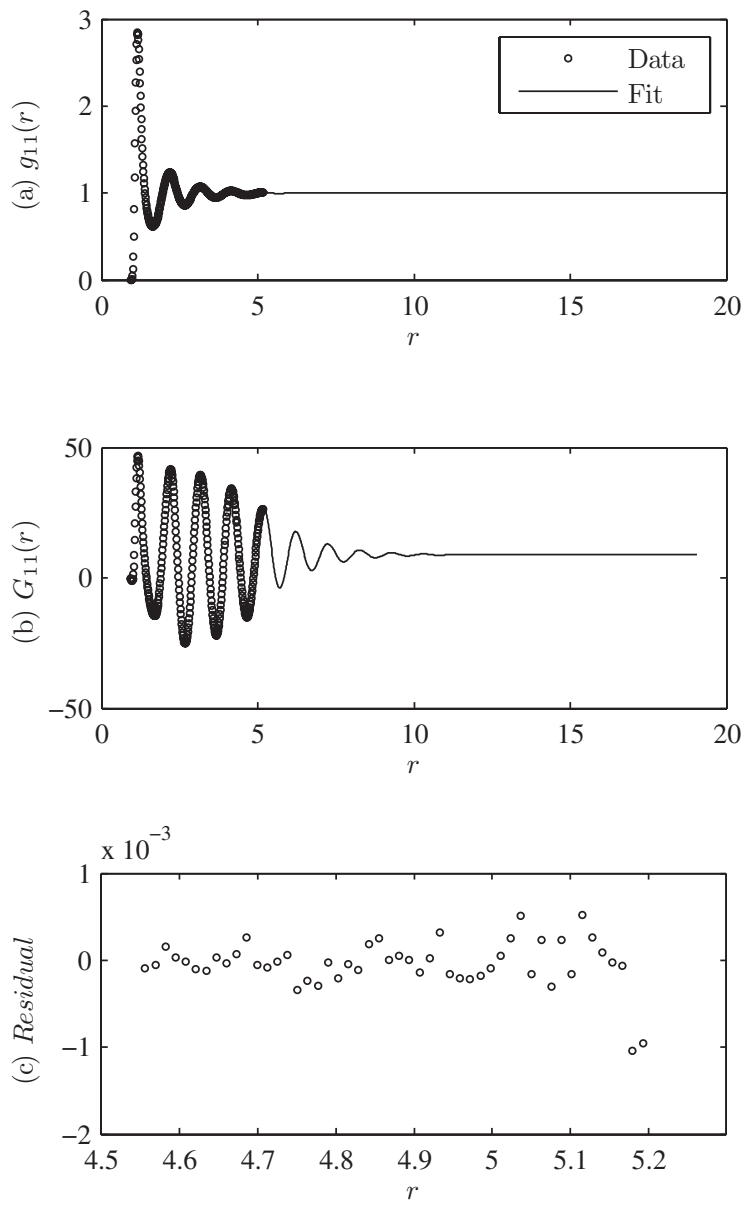

FIG. 7. Equimolar argon-krypton fluid (a) radial distribution function, (b) its integral, and (c) residual of pair distribution and fit for $\mathrm{Kr}-\mathrm{Kr}, n=0.7138$ and $T=0.965$.

\section{Partial specific enthalpies}

To obtain the two component transport coefficients, the G-K integrals are used in Eqs. (31)-(34) which rely upon calculation of the partial specific enthalpies for the fluid. Figures 10 and 11 show enthalpy versus mass for $\mathrm{Kr}$ and $\mathrm{Ar}$. The values of the partial specific enthalpies calculated from the slopes of the lines of best fit are -2.07(1) and -2.33(2), respectively, where the bracketed numbers are the errors in the respective values. The difference $h_{1}-h_{2}$ is the weighting of the contribution from the relevant unprimed phenomenological coefficients when calculating the primed phenomenological coefficients with Eqs. (31)-(34).

\section{Transport coefficients}

Combining the results of the above simulations, we have calculated the transport coefficients shown in Table IV. For comparison with previous work, we also include the mutual diffusion coefficient calculated using the assumption of ideality $D_{m}($ ideal $)$, although this value of the mutual diffusion coefficient is incorrect for equimolar Ar-Kr. Our value of 0.0491(2) compares well with the results of Jolly and Bearman who obtain a value of 0.0489(4). ${ }^{9}$ The sign of $D^{\prime}$ indicates the direction in which a species will migrate. This is
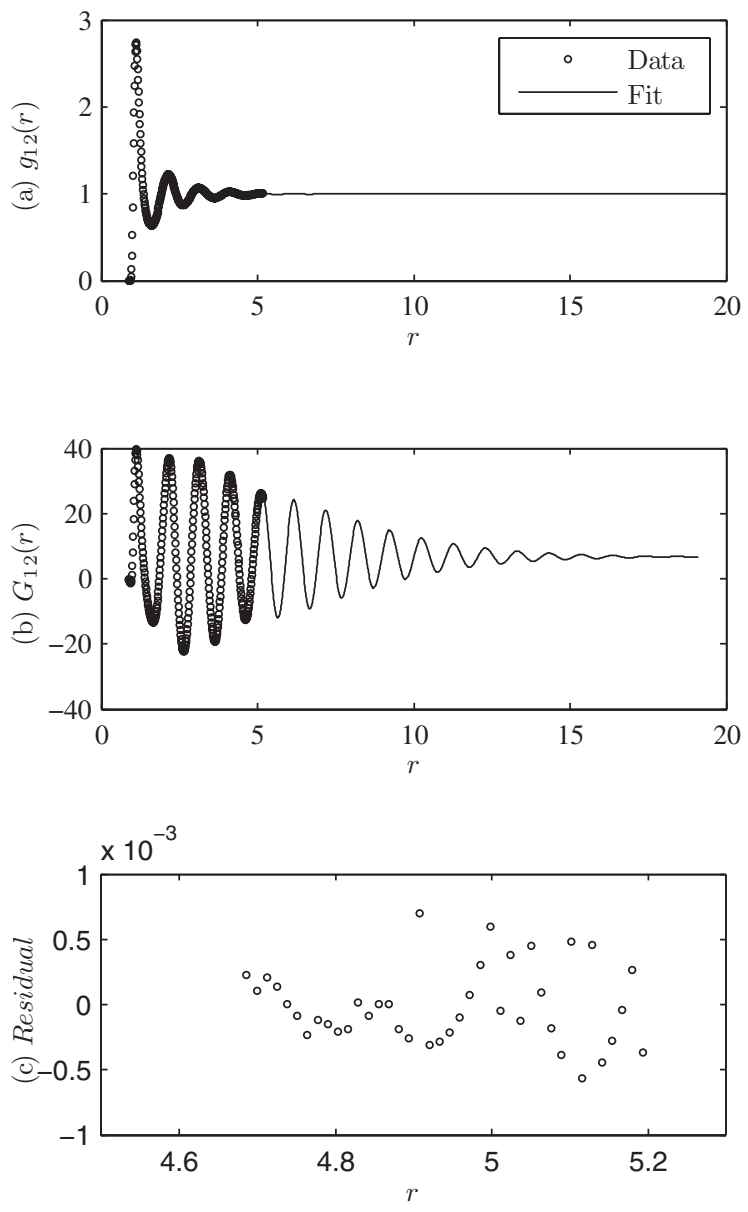

FIG. 8. Equimolar argon-krypton fluid (a) radial distribution function, (b) its integral, and (c) residual of pair distribution and fit for $\mathrm{Ar}-\mathrm{Kr}, n=0.7138$ and $T=0.965$.

positive for the $\mathrm{Kr}$ species. We can compare our result for the thermal diffusion factor to the result presented by Hafskjold, Ikeshoji, and Ratkje ${ }^{16}$ who reported

$$
\alpha_{12}=T \frac{D^{\prime}}{D_{m}} .
$$

In this study, we find a value of 1.6(1) which is comparable to their value of 1.5(1). The method presented in this study requires more computation, but it has the advantage of obtaining the individual contributions due to the thermal diffusion coefficient and mass diffusion coefficient separately.

\section{B. Confined temperature difference systems}

The transport coefficients are used to make a continuum prediction of the temperature dependent concentration profile for a highly confined fluid through Eq. (43). To simulate the confined fluid, two hexagonal close packed, three layer walls are constructed by tethering the centres of mass of 768 Ar particles to lattice sites with a reduced spring force of 57.15. The temperatures of the walls are controlled using Gaussian thermostats at reduced temperatures of 0.915 and 1.015. The fluid is composed of $7680 \mathrm{Ar}$ and $7680 \mathrm{Kr}$ particles sandwiched between the parallel walls. The volume of the channel is set so that the confined fluid density matches the density of the 

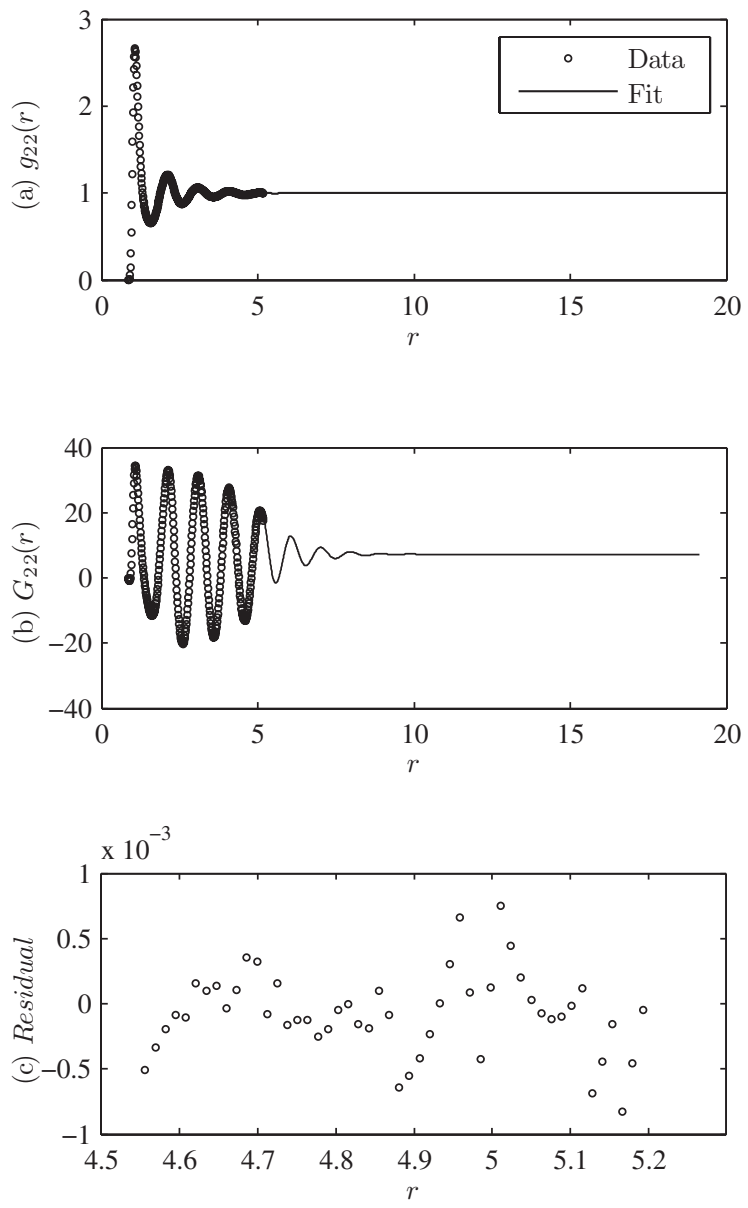

FIG. 9. Equimolar argon-krypton fluid (a) radial distribution function, (b) its integral, and (c) residual of pair distribution and fit for $\mathrm{Ar}-\mathrm{Ar}, n=0.7138$ and $T=0.965$.

TABLE III. Ar-Kr volume integral values of radial distribution functions, $n=0.7138$ and $T=0.965$. The number in brackets is the error in the last decimal place.

\begin{tabular}{lcc}
\hline \hline Species & Index & $G_{a b}$ \\
\hline $\mathrm{Kr}-\mathrm{Kr}$ & $1-1$ & $8.903(1)$ \\
$\mathrm{Ar}-\mathrm{Kr}$ & $2-1$ & $6.891(1)$ \\
$\mathrm{Ar}-\mathrm{Ar}$ & $2-2$ & $7.273(1)$ \\
\hline \hline
\end{tabular}

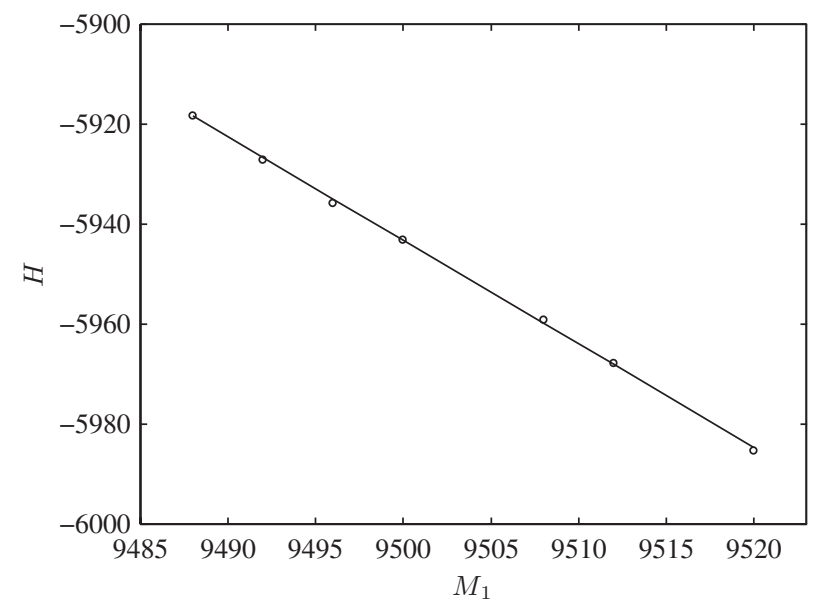

FIG. 10. Enthalpy versus mass of $\mathrm{Kr}, p=8.967$ and $T=0.965$.

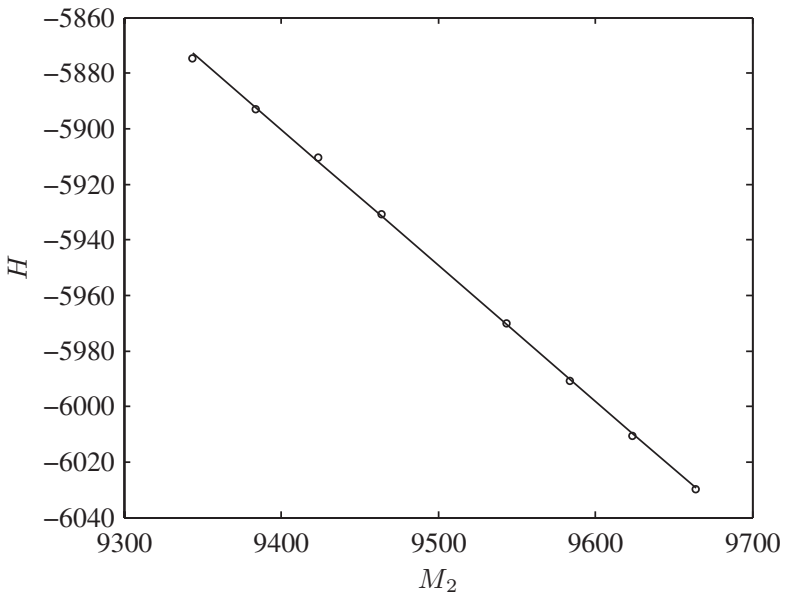

FIG. 11. Enthalpy versus mass of $\mathrm{Ar}, p=8.967$ and $T=0.965$.

bulk fluid for which the transport coefficients have been calculated. Periodic boundary conditions are used in the $x$ and $z$ directions of the fluid to create an effectively infinite length channel.

The temperature and concentration profiles are shown in Figs. 12 and 13, with the predicted concentration profile shown as a solid line. Figure 14 shows a magnified view of the concentration profile. The prediction of Eq. (43) describes the concentration profile in the centre of the channel for the $\mathrm{Kr}$ species reasonably well. For the prediction, we have used a second order polynomial to fit the temperature profile seen in Fig. 12 as the solid line.

The width of the channel, $y$ is measured in lengths of the $\mathrm{Kr}-\mathrm{Kr}$ potential. At a distance of $7 \sigma$ from the colder wall thermostated at $T=0.915$, the concentration of $\mathrm{Kr}$ is 0.765 , and at the same distance from the warmer wall thermostated at $T=1.015$, the concentration is 0.743 . This represents a separation of the $\mathrm{Kr}$ species of approximately $3 \%$ across a distance of $66 \sigma$. Further separation could be achieved by combining larger distances with a greater temperature difference imposed by the walls. However, it is expected that larger temperature differences across the same channel width of $80 \sigma$ would result in a nonlinear concentration profile.

In the region of approximately $7 \sigma$ from the wall at both sides, particle layering becomes a dominant feature. The continuum prediction presented in this work is not intended to account for the concentration and density variations in these regions.

TABLE IV. Transport coefficients for equimolar Ar-Kr, $n=0.7138$ and $T=0.965$.

\begin{tabular}{lcccc}
\hline \hline$D_{m}$ & $D_{m}($ ideal $)$ & $D^{\prime}$ & $\lambda$ & $\eta$ \\
\hline $0.033(1)$ & $0.0491(2)$ & $0.054(4)$ & $4.28(4)$ & $2.581(9)$ \\
\hline \hline
\end{tabular}




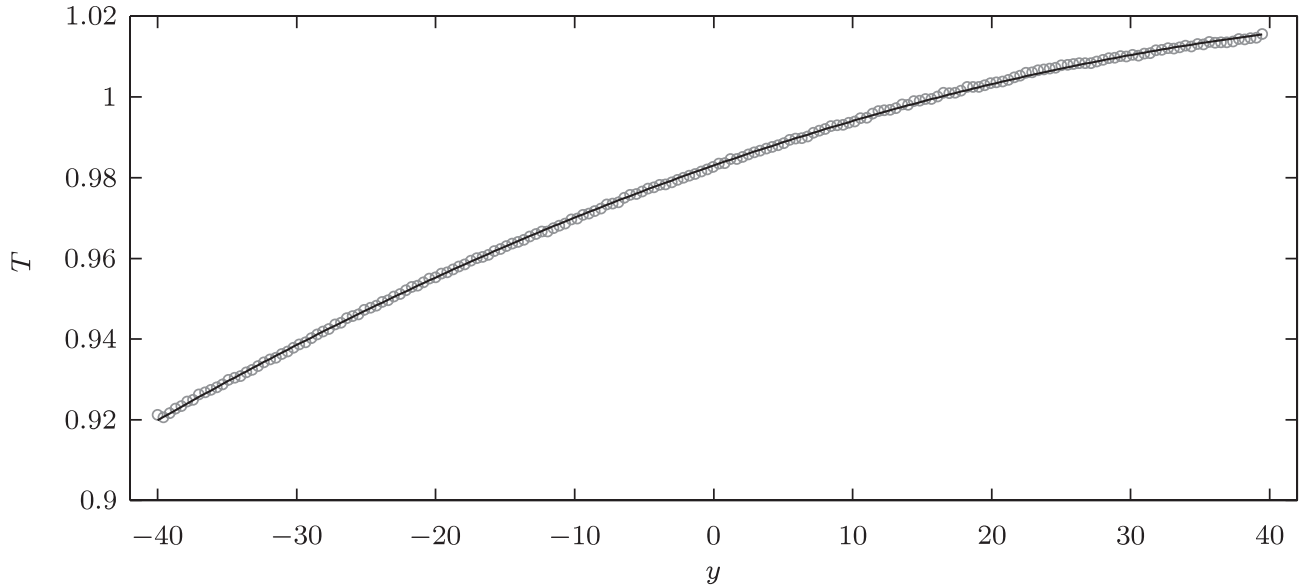

FIG. 12. Equimolar Ar-Kr temperature profile for confined temperature difference system with wall temperatures of $T=0.915$ and 1.015 . A second order polynomial fit is shown as the solid line.

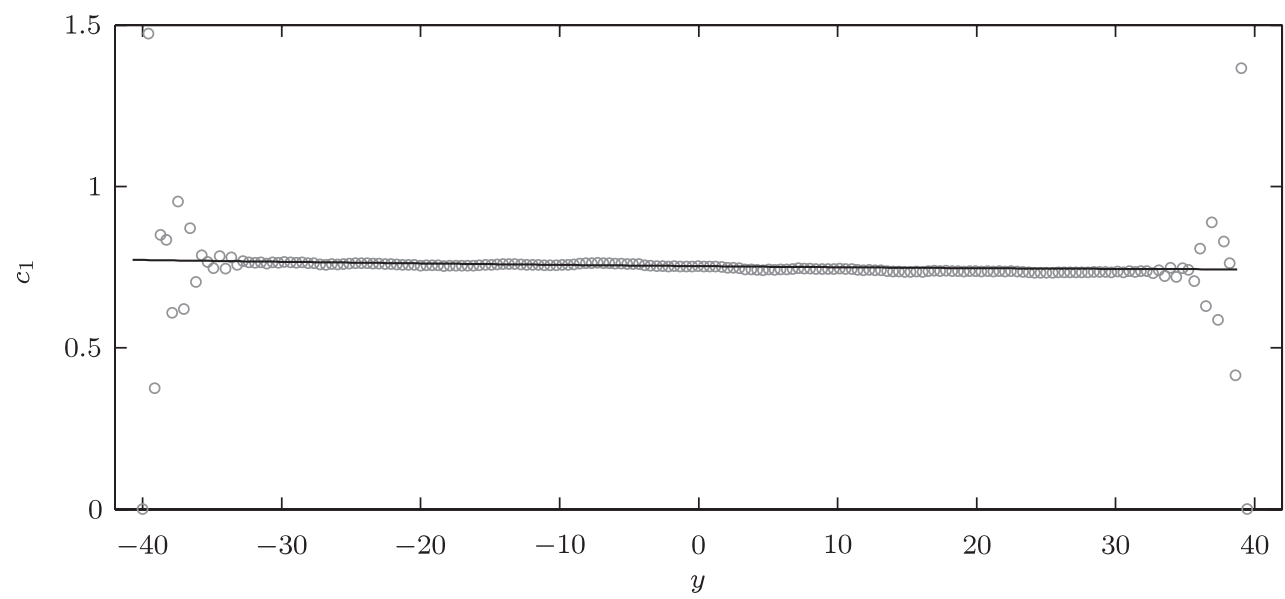

FIG. 13. Krypton concentration profile for confined temperature difference system with wall temperatures of $T=0.915$ and 1.015 .

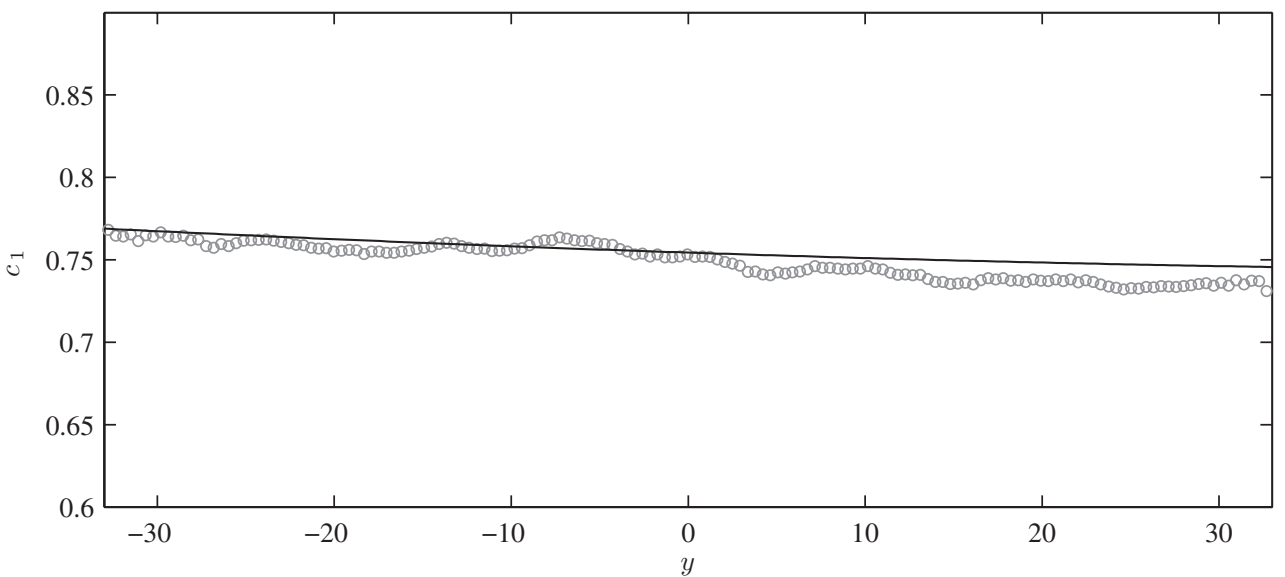

FIG. 14. Magnified $\mathrm{Kr}$ concentration profile for confined temperature difference system with wall temperatures of $T=0.915$ and 1.015 . 


\section{CONCLUSIONS}

We have computed the transport coefficients, specifically the viscosity, mutual diffusion coefficient, thermal conductivity, and thermal diffusion coefficient of an equimolar Ar$\mathrm{Kr}$ mixture. Our results for the unprimed phenomenological coefficients agree with those computed previously from the Green-Kubo relations. Using the relationship between the unprimed and primed phenomenological coefficients, we obtained values of the transport coefficients that also agree with those computed independently by others. The value that we found for the mutual diffusion coefficient indicates that the ideal solution assumption is not valid for this mixture. Using this method, we have successfully obtained accurate and separate values for the mutual diffusion and thermal diffusion coefficients, which have previously only been calculated in the form of their ratio as the thermal diffusion factor.

Using these coefficients, we have made a reasonable prediction of the temperature dependent concentration profile for confined fluids experiencing a temperature gradient imparted on the fluid through the walls. By successfully accounting for the effects of thermal diffusion in the confined fluid, we have shown how the effect can be used in the separation of an equimolar Ar-Kr mixture. The combined effects of heat and mass diffusion have the net effect that the larger $\mathrm{Kr}$ particles migrate to the cooler region of the fluid, creating an increased concentration of approximately $3 \%$.

In a future publication, we will present results for a model colloidal fluid, in which a much smaller temperature difference results in a greater separation of the larger species, and for which the temperature profile can be predicted with linear non-equilibrium thermodynamics.

\section{ACKNOWLEDGMENTS}

We wish to acknowledge the support of the Australian Government through the Australian Postgraduate Awards (APA) scholarship, and the Victorian Partnership for Advanced Computing (VPAC) for the use of their highperformance computing facilities and the Australian Research Council for support under the Discovery Projects scheme (project number DP120102976).

${ }^{1}$ R. C. Jones and W. H. Furry, "The separation of isotopes by thermal diffusion," Rev. Mod. Phys. 18, 151 (1946).

${ }^{2} \mathrm{~K}$. Clusius and G. Dickel, "Neues verfahren zur gasentmischung und isotopentrennung," Naturwissenschaften 26, 546 (1938).
${ }^{3}$ M. M. Bou-Ali, O. Ecenarro, J. A. Madariaga, C. M. Santamaria, and J. J. Valencia, "Thermogravitational measurement of the Soret coefficient of liquid mixtures," J. Phys.: Condens. Matter 10, 3321 (1998).

${ }^{4}$ S. Wiegand, "Thermal diffusion in liquid mixtures and polymer solutions," J. Phys.: Condens. Matter 16, R357 (2004).

${ }^{5}$ R. Piazza and A. Parola, "Thermophoresis in colloidal suspensions," J. Phys.: Condens. Matter 20, 153102 (2008).

${ }^{6}$ J. W. Nichols, S. G. Moore, and D. R. Wheeler, "Improved implementation of Kirkwood-Buff solution theory in periodic molecular simulations," Phys. Rev. E 80, 051203 (2009).

${ }^{7}$ P. Krüger, S. K. Schnell, D. Bedeaux, S. Kjelstrup, T. J. H. Vlugt, and J.-M. Simon, "Kirkwood-Buff integrals for finite volumes," J. Phys. Chem. Lett. 4, 235 (2013).

${ }^{8}$ K. Gaylor, I. Snook, and W. van Megen, "Comparison of Brownian dynamics with photon correlation spectroscopy of strongly interacting colloidal particles," J. Chem. Phys. 75, 1682 (1981).

${ }^{9}$ D. L. Jolly and R. J. Bearman, "Molecular dynamics simulation of the mutual and self diffusion coefficients in Lennard-Jones liquid mixtures," Mol. Phys. 41, 137 (1980).

${ }^{10} \mathrm{M}$. Schoen and C. Hoheisel, "The mutual diffusion coefficient $\mathrm{D}_{12}$ in binary liquid model mixtures: Molecular dynamics calculations based on LennardJones (12-6) potentials," Mol. Phys. 52, 33 (1984).

${ }^{11} \mathrm{~S}$. de Groot and P. Mazur, Non-Equilibrium Thermodynamics (Dover Publications, New York, 1984).

${ }^{12}$ A. Perronace, G. Ciccotti, F. Leroy, A. H. Fuchs, and B. Rousseau, "Soret coefficient for liquid argon-krypton mixtures via equilibrium and nonequilibrium molecular dynamics: A comparison with experiments," Phys. Rev. E 66, 031201 (2002)

${ }^{13}$ D. MacGowan and D. J. Evans, "Heat and matter transport in binary-liquid mixtures," Phys. Rev. A 34, 2133 (1986).

${ }^{14}$ S. Sarman and D. J. Evans, "Heat-flow and mass diffusion in binary Lennard-Jones mixtures," Phys. Rev. A 45, 2370 (1992).

${ }^{15} \mathrm{~N}$. Galamba, C. A. Nieto de Castro, and J. F. Ely, "Equilibrium and nonequilibrium molecular dynamics simulations of the thermal conductivity of molten alkali halides," J. Chem. Phys. 126, 204511 (2007).

${ }^{16}$ B. Hafskjold, T. Ikeshoji, and S. K. Ratkje, "On the molecular mechanism of thermal diffusion in liquids," Mol. Phys. 80, 1389 (1993).

${ }^{17}$ D. Evans and G. Morriss, Statistical Mechanics of Nonequilibrium Liquids (Academic Press, London, 1990).

${ }^{18}$ R. Vogelsang, C. Hoheisel, P. Sindzingre, G. Ciccotti, and D. Frenkel, "Computation of partial enthalpies of various Lennard-Jones model mixtures by NPT molecular-dynamics," J. Phys.: Condens. Matter 1, 957 (1989).

${ }^{19}$ P. Sindzingre, G. Ciccotti, C. Massobrio, and D. Frenkel, "Partial enthalpies and related quantities in mixtures from computer-simulation," Chem. Phys. Lett. 136, 35 (1987).

${ }^{20}$ J. G. Kirkwood and F. P. Buff, "The statistical mechanical theory of solutions .1," J. Chem. Phys. 19, 774 (1951).

${ }^{21}$ J. P. Hansen and I. R. McDonald, Theory of Simple Liquids (Elsevier Science, 2006).

${ }^{22}$ P. J. Daivis and D. J. Evans, "Comparison of constant-pressure and constant volume nonequilibrium simulations of sheared model decane," J. Chem. Phys. 100, 541 (1994).

${ }^{23} \mathrm{~S}$. Duhr and D. Braun, "Thermophoretic depletion follows Boltzmann distribution," Phys. Rev. Lett. 96, 168301 (2006)

${ }^{24}$ S. Duhr and D. Braun, "Optothermal molecule trapping by opposing fluid flow with thermophoretic drift,” Phys. Rev. Lett. 97, 038103 (2006). 